Volume 6, Issue 2, April 2021, pp. $111-127$

DOI: $10.23917 /$ jramathedu.v6i2.11130

p-ISSN: 2503-3697, e-ISSN: 2541-2590

\title{
Mathematics education undergraduates' personal definitions of the notion of angle of contiguity in Kinematics
}

\author{
Zakaria Ndemo ${ }^{1 *}$, David Mtetwa ${ }^{2}$ \\ ${ }^{1}$ Department of Science and Mathematics Education, Bindura University of Science Education, Zimbabwe \\ ${ }^{2}$ Department of Science and Mathematics Education, University of Zimbabwe, Zimbabwe \\ *Corresponding author: zndemo@gmail.com
}

\begin{tabular}{l}
\hline ARTICLE INFO \\
\hline Article history: \\
Received: 12 July 2020 \\
Revised: 17 December 2020 \\
Accepted: 23 December 2020 \\
Published online: 24 March \\
2021 \\
Published regularly: April \\
2021
\end{tabular}

Keywords:

Mathematical definition,

personal concept definition,

angle of contiguity, student teachers

\section{ABSTRACT}

The concept of a mathematical definition causes severe difficulties among students during problem solving and proving activities. Students' difficulties with the use of mathematical definitions often arise from the fact that students are often given those definitions instead of constructing them. With the aim of developing an understanding of the kinds of student teachers evoked concept images of the notion of angle of contiguity, a qualitative case study was conducted at one state university in Zimbabwe. Purposive sampling was used to select 28 mathematics undergraduate student teachers who responded to a test item. Qualitative data analysis was guided by ideas drawn from the theoretical framework of Abstraction in Context and idea of imperative features of a mathematical definition. Student teachers written responses revealed that student teachers personal concept definitions consisted of ambiguous and irrelevant formulations that did not capture the essence of the idea of the angle of contiguity. In some cases their responses were not consistent with the definition of the angle of contiguity. Although there were a few instances of adequate descriptions of the concept, (8 out of 32) these and the inadequate descriptors elicited can contribute significantly towards efforts intended to improve mathematics instruction. Improved mathematics instruction will lead to enhanced conceptualizations of mathematics concepts.

(C) 2021 Universitas Muhammadiyah Surakarta

\section{Introduction}

The concept of a mathematical definition is essential in developing mathematical knowledge and hence central to the learning of mathematics (Gilboa, Kidron \& Dreyfus, 2019). However, despite mathematical definitions commanding a central position in

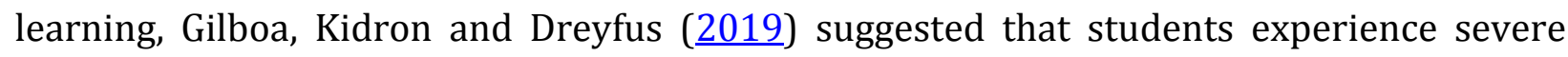
difficulties with mathematical definitions that include the following. Gilboa, Kidron and Dreyfus found that students have a weak command of the nature and function of 
mathematical definitions, and are unable to apply definitions in solving related problems. Students' difficulties in acquiring mathematical knowledge are caused by their lack of meta-mathematical knowledge Carlson and Bloom (2005). They define meta-mathematical knowledge as monitoring of thought process during problem solving. Meta-mathematical knowledge may include being able to differentiate between a definition and theorem and awareness of one's weak grasp of counter-argumentation in proof and proving activities.

Constructing definitions is a basic component of the process of building mathematical knowledge. Definitions are essential in developing mathematical knowledge because they express the properties that characterize the objects of a given mathematical idea and then relate them in a network of relationships (Ubuz, B., Dincer, \& Bülbül, 2013: p. 313). When composing definitions, meditating on relationships established fosters an individual's problem solving abilities. Hence, the ability to define a concept has important consequences for problem solving and mathematics learning (Edwards \& Ward, 2004). However, research studies (CadawalladerOlsker, 2011; Harel \& Sowder, 1998; Zaslavsky \& Shir, 2005) have focused on aspects such as requesting students to identify examples and non-examples of a given concept from statements supplied by the researchers.

Selden and Selden (2003) reported that instruction at undergraduate level has followed the format of definition $\rightarrow$ theorem $\rightarrow$ proof, with much emphasis on attempts to prove theorems using definitions supplied and other previously proven results. Gilboa, Kidron and Dreyfus ( $\underline{2019}$ ) noted that little is known about how students come to know about definitions because they are rarely requested to construct mathematical definitions. Thus, although mathematics educators request students to use definitions to solve problems and prove mathematical conjectures they rarely focus on how those definitions are constructed. There is, therefore, scarcity of studies that investigate students thought processes as they engage on definition tasks.

In response to a dearth on studies on student teachers abilities to construct definitions, the current study focuses on investigating students thinking about concepts in Theoretical Mechanics. More specifically, the study involved a request for undergraduate mathematics education students to define the concept of the angle of contiguity - a key concept in the Kinematics section of Theoretical Mechanics. The aim of the present study is to explore the kinds of student teachers personal concept definitions. A personal concept definition is a reconstruction of the definition of the concept at stake by a student. In other words a personal concept definition is a representation or explanation of an individual concept image (Nurwahyu, 2014).

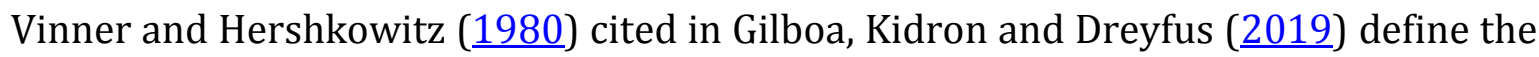
term concept image as "a set of all pictures that have ever been associated with the concept ... (and) a set of properties that have ever been associated with the concept" (p. 422). Concept images reveal how students interpret a concept (Jamila, Suryadi \& Priatna, 2019). The students' personal concept definitions constitute students evoked concept images during those moments they construct the definition of a concept. The intent was to develop an understanding of the kinds of in-service teacher informants' personal definitions of the notion of the angle of contiguity. Developing such an understanding can contribute towards efforts to stimulate a refinement in students' conceptions of the 
mathematical idea and related ideas. For instance, a firm grasp of the definition of angle contiguity can allow students to construct proof of the theorem: the projection of the acceleration vector on the normal to the path is equal to the square of the numerical value of the velocity divided by the radius of curvature. In symbols we have: $a_{n}=\frac{v^{2}}{R}$, where $a_{n}$ denotes the component of the acceleration vector along the normal axis, $v$ refers to the velocity and $R$ denotes the radius of curvature. Furthermore, improved conceptions of the notion of contiguity can enhance students reasoning when they engage with problems dependent on the concept. To gain insights into the kinds of student thoughts as they engage with tasks involving formulating definitions, the following research question posed: What are the kinds of undergraduate students evoked concept images with respect to the notion of angle of contiguity in Kinematics?

By focusing on this research question, the study aims to explicate students reasoning. The point is that by examining informants' evoked concept images we aim to generate insights into the kinds of visual representations, and a collection of impressions developed from student teachers experiences with the concept of the angle of contiguity. Such representations can then provide some sense of the kinds of students' examples or nonexamples of the definition of the concept.

The significance of the study pertains to the fact that the teacher acts as a facilitator who knows the definition construction path. Thus, if the student teacher's command of the knowledge of one of the key concepts in Theoretical Mechanics is weak then she/he would not be able to foster in her/his future learners' abilities to construct definitions effectively. Hence, it is imperative to address the research question posed about informants' concept images in order to explore mathematics education undergraduates' competences at constructing definitions of mathematical concepts. We now focus on theoretical underpinnings that guided the study.

First, the notion of angle of contiguity which is central in the study of geometrical properties of motion of a rigid body at undergraduate level was examined. They are treated in a branch of Theoretical Mechanics called Kinematics. The concept of angle of contiguity is an essential building block in developing the concept of instantaneous acceleration of a body. The notion is especially useful when analyzing the projection of the acceleration vector on the normal axis the Natural trihedron axes as illustrated in Figure 1 which follows.

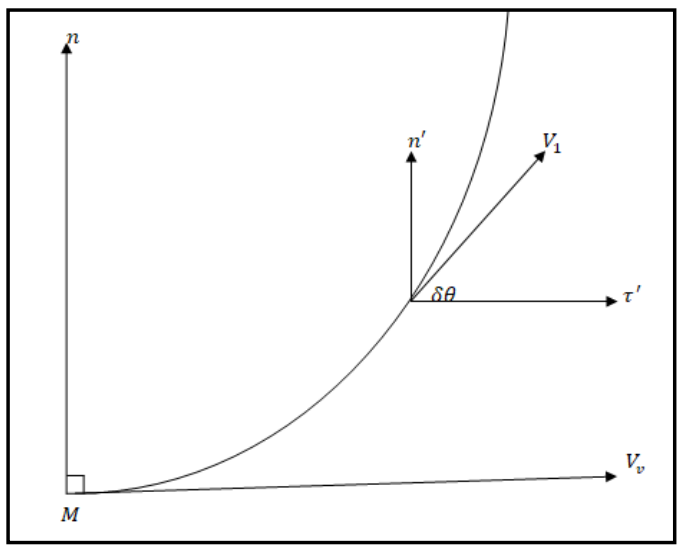

Figure1. The concept of angle of contiguity 
Figure 1 shows a particle at $\underset{V_{1}}{M^{\prime}}$ after it had moved from its initial position $M$. From Figure 1, the angle $\partial \theta$, the velocity $\vec{v}_{1}$, makes with the tangential axis is called the angle of contiguity. The angle of contiguity illustrated in Figure 1 is essential in developing the concept of curvature in Kinematics. The concept of curvature in Kinematics is defined as the limit of the quotient $\frac{\delta \theta}{\delta s}$ as $\delta s \rightarrow 0$. The concept of curvature just described is an important requisite idea to the proof of theorem: the projection of the acceleration vector on the normal to the path is equal to the square of the numerical value of the velocity divided by the radius of curvature. Further, we observe that curvature is the inverse of the radius of curvature $R$, that is, $\lim _{\delta s \rightarrow 0} \frac{\delta \theta}{\delta s}=\frac{1}{R}$. The relationship between the two notions of curvature and radius of curvature is an essential building block in the derivation of the mathematical object $\alpha_{n}=\frac{v^{2}}{R}$.

Second, the current study draws from Davydov's (1992) notion of Abstraction in Context (AiC) and Zaslasvky and Shirs (2005) ideas of roles and features of mathematical definitions. Accordingly, in this section we begin by explaining the AiC notion and then examine the roles and features of mathematical definitions in the context of this study.

According to Davydov (1990), constructing a mathematical definition involves abstraction. Drawing from Davydovs ideas Gilboa, Kidron and Dreyfus ( $\underline{2019})$ write that abstraction starts with a simple undeveloped idea which will lead to a consistent and elaborate final form. The construct of Abstraction in Context (AiC) is a tool for investigating processes of constructing knowledge. Constructing knowledge involves reorganizing vertically previous mathematical objects and this vertical reorientation of existing knowledge elements captured in the description of the AiC notion illustrates the idea that previous knowledge components serve as building blocks in the process of constructing a definition. For instance, in the context of the current study the notion of a tangent to a curve with its restrictions described earlier in our introductory remarks are key knowledge elements in constructing the definition of the angle of contiguity. Furthermore, the concept of instantaneous acceleration defined by $\alpha=\lim _{\delta t \rightarrow 0} \frac{\delta v}{\delta t}=\frac{d v}{d t}$, where $v$ denotes the velocity function and $d t$ is a differential time element is needed to define the concept of angle of contiguity.

The AiC process proceeds through three stages: the need for a new construct, the construction stage and the consolidation stage (Gilboa et al., 2019). The first stage (the need for a new construct) initiates the abstraction process. A students interest in the topic or problem being grappled with can often motivate him/her to compose a definition. This was an important idea that informed the data collection procedure (see the Methodology section). According to Davydov (1990) the construction stage consists of three epistemic actions for knowledge generation: recognizing (R), building (B), and constructing (C). In this sense, the AiC theoretical framework is composed of three observable actions for knowledge generation. First, the epistemic action, R, refers to a stage whereby a learner recognizes previous knowledge elements pertinent to the definition to be constructed. In the current study, the relevant previous knowledge elements to the concept of angle of contiguity include mathematical ideas about tangents, the concept of binormal, normal and 
tangential axes as three mutually perpendicular directions. The second epistemic action, B, is whereby a learner acts on the recognized pieces of knowledge. Thus, student teachers operating at this epistemic level are expected to reflect and establish connections between existing knowledge elements (curve or path, derivative, tangent, trihedron axes) in order to make sense of the notion of angle of contiguity. Finally, the third epistemic actions, at $C$ (constructing) level involve relating and interweaving the $\mathrm{R}$ and $\mathrm{B}$ epistemic actions for the purpose of composing the required mathematical definition. The learner then communicates the concept constructed either verbally (by mentioning it) or in written form. Finally, the AiC process has a consolidation stage whereby a learner applies what has been constructed to compose other definitions or proving other theorems (Gilboa et al., 2019). The RBC epistemic actions of the construction stage formed the basis for data analysis of the study together with the notion of roles and imperative features of a definition which is the focus of the next section.

The question of how students develop their personal concept definitions is now discussed in this section. First, we consider the notion of a definition of a mathematical concept. Zaslavsky and Shir (2005, p.142) write that "The mathematical term definition is one of a handful of meta-mathematical terms (others include axiom, lemma, proposition, theorem, corollary, proof), terms which serve to indicate the purported status and function of elements of written mathematics."

The phrase "purported status and function" captured in this description of a mathematical definition points to the fact that a concept definition should be a complete description of the behaviour, structure or properties of a focal mathematical idea (Wilkerson \& Jerde, 2011). In other words, a mathematical definition should include all instances of the focal mathematical idea. Hence, adequacy of a mathematical definition of a concept is a key requirement when defining it. Zaslavsky and Shir (2005) suggest that students usually interact with examples and non-examples of a concept hand in hand with the definition of that concept. Zaslasky and Shir observe that students are usually exposed to extractions from textbooks. A worrisome scenario in mathematics instruction is that a word-for-word replica of mathematical definitions in polished form from existing literature sources is supplied by teachers to the students. Thus, students are rarely requested to construct definitions but rather students are expected to use definitions given by teachers to do the following. First, definitions are used to introduce the objects of a given theory and capture the essence of a focal mathematical idea by conveying its characterizing properties (Mariotti \& Fischbein, 1997). Second, definitions are used to form other concepts using given definitions as fundamental building blocks. Finally, they are employed in proving and problem solving activities (Dreyfus, Hoyles, Guedet, \& Krainer, 2014).

In addition to the idea of inadequate personal concept definitions discussed thus far, a student personal concept definition also relies on imperative features of a mathematical definition (van Dormolen \& Zaslavsky, 2003). Thus, if a statement purported to be a definition of a given concept does not satisfy one of the imperative features then it constitutes another kind of a non-example of that mathematical concept. The imperative features of a student personal definition relate to the following requirements suggested by Zaslavsky and Shir (2005). 
A students' personal concept definition must be non-contradicting. By being noncontradicting, Zaslavsky and Shir ( $\underline{2005}$ ) explain that the constituent elements of a definition must reveal consistency to allow them to coexist. For example, in Elementary Geometry, the assertion: an obtuse angled triangle can be right angled is not noncontradicting. The elements, right and obtuse angles cannot coexist because once a triangle contains an obtuse angle it implies that the two remaining can only be acute angles. Another imperative feature of a mathematical definition is that it needs to be nonambiguous. In other words, a formulation of a concept definition must be uniquely interpreted. The underlying idea is that an expression purported to constitute a definition must not lead to different inferences of its meaning.

Yet another imperative feature of a mathematical definition relates to the hierarchical nature of a mathematical definition. A hierarchical definition is formulated on the basis of previously developed basic ideas. For example, the ideas of tangents, binormal axis and the notion of velocity as a vector are prerequisite ideas to the concept of the angle of contiguity in Theoretical Mechanics. Fourth, the meaning of a personal concept definition must be invariant under a change of representation systems or reference theory. For instance, the visual representation of the angle of contiguity must discern the same meaning as that conveyed by the narrative form (Zaslavsky \& Shir, 2005).

Student teachers constructions of the definitions of the angle of contiguity could be evaluated on the basis of the notion of minimality. A mathematical definition is considered to be minimal if it is economical with no superfluous, unnecessary formulations. Hence, a minimal definition must consist of information that is strictly necessary to reveal the adequate mathematical structure, behaviour or relationships or properties shared by the focal mathematical ideas constituting the definition of that concept (Van Dormolen \& Zaslavsky, 2003)

\section{Research Methods}

An exploratory case study design was used to provide insights to the kinds of students personal concept definitions of the notion of angle of contiguity (Punch, 2005; Yin, 2009). This design was considered because it allowed prolonged interaction with the participants (Baxter \& Jack, 2010; Maxwell \& Mittapalli, 2010). The study took place during a 15 week long semester during which the first author of this article provided tuition to the undergraduate mathematics education students involved in this study. Prolonged engagement allowed the researchers to evaluate the kinds of epistemic states of the construction stage of the AiC construct namely: recognizing (R), building (B) and the (C) level whereby students were anticipated to relate $\mathrm{R}$ and $\mathrm{B}$ elements in order to compose the definition of the angle of continuity.

The focus of the study was on the first two stages of the AiC theoretical framework. Purposive sampling technique was used to select 28 mathematics education undergraduate student teachers who had enrolled for the in-service Bachelor of Science Education (BSc Ed) Degree. The student teachers were in their final semester of a three year course. The (BSc Ed) degree programme is intended to capacitate Diploma in Education holders to teach mathematics up to Advanced level. The diploma holder must have taught 
mathematics at secondary school level for at least 2 years. So, the BSc Ed degree programme of study is some form of Continuing Professional Development (CPD) initiative in Zimbabwe. The structure of BSc Ed degree programme is as follows.

Students undergoing CPD study content and professional courses. During year 1, students study 8 subject matter and 4 professional courses. Subject content materials are drawn from the following learning areas: Calculus, Linear Algebra, Differential Equations and Numerical Methods. The professional component of the undergraduate studies includes modules such as Foundations in Science Education and Research Methods. Foundations in Science Education involve the study of psychological, sociological and philosophical aspects of the professional curriculum. The Research Methods module covers issues in quantitative and qualitative research paradigms and descriptive and inferential statistics concepts. It is intended to prepare students for research projects in the final year. During the second year, students also study 8 subject content courses focusing on Real Analysis, Optimization, Probability Theory and Statistics. Three professional courses are studied in year 2: History and Philosophy of Mathematics, Pedagogical Content Knowledge and a preparatory course for Teaching Practicum.

During the final year of study learning areas that include Partial Differential Equations and Fourier Series, Complex Variables and Mechanics are covered. For the professional component student teachers do a research project on teaching and learning of school mathematics. As indicated earlier the first author taught Mechanics course and thus it served as a setting for the study. It is, therefore, imperative to describe subject content material for the Mechanics course.

Mechanics at undergraduate level covers three broad learning areas: Statics, Kinematics and Dynamics. In Statics, the concept of a force is central. Here the equilibrium conditions of a material body under the action of coplanar concurrent forces are treated. The distinction between a free and a constrained body is captured by the axiom of constraints. Other fundamental concepts treated at this level include the concepts of resolution and composition of force systems. In addition, the learning area of Statics also includes the concepts of kinetic and static friction as well as the notion of angle of friction.

Dynamics of rigid bodies covers such aspects as the impulse-momentum equation, translational and rotational motion of a rigid body. The concepts covered in this branch of mechanics include the notion of angular momentum and a treatment of theorems on the conservation of both angular and linear momentum in a conservative force field. In addition, the concept of impact is developed with a distinction explicated between direct and oblique central impact. The coefficient of restitution is introduced and used to explain notions of perfectly elastic and inelastic impact. The Carnot Theorem of energy losses in a perfectly elastic collision is introduced and proved. Other ideas covered include the concept of center of mass, Parallel Axis Theorem and a treatment of the concepts of virtual displacement and virtual work.

Kinematics treats concepts of rectilinear and curvilinear motion. Kinematics also deals with methods of describing motion namely: natural, vector and coordinate. Key geometric properties of motion including path, velocity and acceleration are treated. The notions of instantaneous velocity and acceleration are introduced and developed in this 
learning area. The concept of natural trihedron axes is introduced and the resolution of the total acceleration leads to the ideas of normal and tangential acceleration. The notion of angle of contiguity which is central to the development of the concept of normal acceleration became the focus of this study.

\section{Data collection procedure}

As indicated in preceding subsection of the methodology section, the context for the study consisted of a 15 week long semester during which Mechanics concepts were taught to undergraduate mathematics education students. This setting (mathematics lectures) constituted a suitable environment in which discussions were held of examples and nonexamples of concepts in Mechanics. During the instruction the concept of the angle of contiguity was discussed. However, the authors hasten to say a formal definition of the idea was not given to the participants. Rather a visual representation was made when proving the theorem that characterizes the projections of the acceleration vector on the trihedron axes. Guided by stage 1 of the AiC theoretical framework, the first author provoked discussions which would arouse the need for the definition.

The objectives of the teaching instruction were articulated to the informants. For instance, one of the instructional objectives was that students should be able to define the concept of curvature which is defined as $\lim _{\Delta t \rightarrow 0} \frac{\Delta s}{\Delta \theta}$ where $\Delta s$ denotes a differential element in displacement and $\Delta \theta$ is the angle of contiguity - an essential ingredient in the proving

the theorem: $\alpha_{n}=\frac{v^{2}}{R}$. That way students were put in a situation in which they were provoked to search for the concept definition in a sense suggested by Kidron (2011). Data collection took place during week 8 of the semester when students had covered learning material on Kinematics.

To collect data test scripts were distributed to student teachers. The informants then responded to the test items individually under the supervision of the first author who played a researcher-tutor dual role. Students' written response to one of the items in a formative test constituted data for this study. One of the test items was framed as: 2 (a) Define the following terms (i) instantaneous velocity, (ii) angle of contiguity.

The intent was to generate independent students' thoughts. The researchers decided to focus on students written productions to item 2 (a) (ii). The data collection technique was deemed strategic since it allowed the authors to elicit authentic student productions as they would be striving to earn high marks in the formative test. However, for the purpose of this study, the focus was not on marks but rather on thinking illuminated in student teachers written productions of the concept definition. Furthermore, the focus of the study was on in-depth study of a single item to develop a profound understanding of the students' abilities to compose definitions.

\section{Data analysis}

We first produced two sets of the data. We then applied content analysis to the qualitative data. We then met to discuss emerging categories of data. The instances where we differed on kinds of emerging themes one of the two of us would be allowed to justify themes until a consensus was reached. Content analysis technique suggested by Berg 
(2009) was applied to students written responses to the task. The three epistemic states of the $\mathrm{AiC}$ construct and the imperative features of a definition formed the lens through which students written efforts were scrutinized. The theoretical frameworks: the notion of Abstraction in Context (AiC), the imperative features of a definition and the notion of a concept image formed a theoretical lens (Creswell, 2014), for examining the informants written responses to the definition task posed. For instance, student teachers written response was examined to determine the nature epistemic actions employed. Further, content analysis technique (Berg, 2009) was used to categorize written responses according to imperative features of a definition. Hence, data analysis was accomplished by mapping students written responses to epistemic actions of the construction stage of the AiC theoretical framework (Gilboa et al., 2019). Further, the informants written productions were categorized as examples or non-examples of the concept definition depending on the level of adequacy. In addition, textual data of the test item were scrutinized through the lenses of the imperative features of a concept definition: noncontradicting, non-ambiguous, whether meaning remains invariant with a change of representation, hierarchical nature of concept definitions (Zaslavsky \& Shir, 2005). Further, textual data were examined to determine whether the condition of minimality of a concept definition was not violated (Punch, 2005; Zaslavsky \& Shir, 2005). Content analysis of data also yielded categories which could not be accounted for in terms of elements of $\mathrm{AiC}$ construct and imperative features of a mathematical definition. This category was called "OTHER" and a possible explanation for the existence of this category was suggested. In-vivo codes were used to support inferences drawn from the data (Corbin \& Strauss, 2008). Next, the researchers present and discuss the study findings.

\section{Results and Discussion}

\section{Emerging categories of student teachers personal concept definitions}

Content analysis technique suggested by Berg (2009) was applied to students written responses to the task. The three epistemic states of the AiC construct and the imperative features of a definition formed the lens through which students written efforts were scrutinized. In this section, we present and discuss categories of students responses formed from content analysis of data. An overall picture of the data reduction process is first given in Table 1. However, we emphasize that there was no one to one mapping of written productions. For instance, some students had both graphical and narrative forms of the definitions of the angle of contiguity. Hence, while there were 28 informants the total of the frequencies of the categories was 32 which is greater than 28 as illustrated in Table 1 which follows.

Table 1 reveals that only 8 out of 32 responses were adequate descriptions of the notion of angle of contiguity. Eight out of 32 of students' written productions revealed lack of consistency between graphical representations and the narrative (verbal) forms of students' personal concept definitions. The same table shows that 6 out of 32 written efforts were ambiguous descriptions of the notion of angle of contiguity. Finally, it can be discerned from the same Table 1 that 4 out of 32 written responses could not be classified 
according to imperative features of a personal concept definition. Next, the researcher exemplifies and discusses each category of students' response in Table 1.

Table 1

Student teachers personal concept definitions of the angle of contiguity $(n=28)$

\begin{tabular}{lc}
\hline Category & frequency \\
\hline Adequate (AD) & 8 \\
Ambiguous (AMB) & 6 \\
Invariant under change of representation (IUCR) & 8 \\
Blank (B) & 6 \\
OTHER & 4 \\
Total & 32 \\
\hline
\end{tabular}

\section{Category: Adequate student teacher personal definitions}

This category consisted of responses which were complete descriptions of the mathematical structure and relationships shared by pertinent ideas to the notion of angle of contiguity (Ubuz, Dincer \& Bübül, 2013; Wilkerson \& Jerde, 2011). Typical examples of students efforts in this category are now produced.

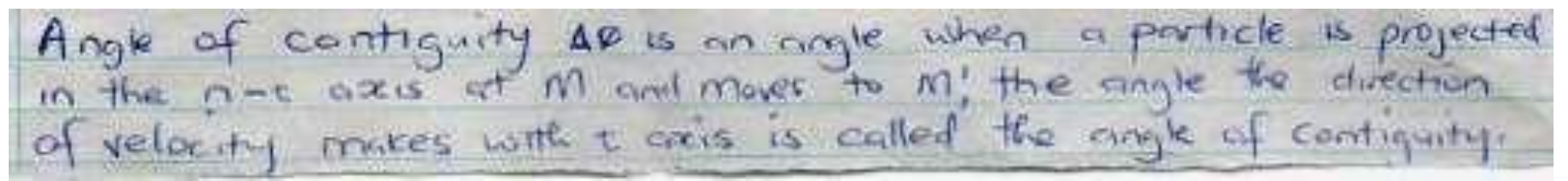

Figure 2. Geofrey's concept definition

First, we noted that Geofrey used the correct notation $\Delta \theta$ to convey the crucial property that the angle of contiguity represents an infinitesimal change in the angle as the particle was displaced from position $M$ to $M^{\prime}$. Fundamental ideas such as the notion of tangent as representing the velocity, the tangential axis denoted by $\tau$ axis were stated and the relationship: "the angle the direction of velocity makes with the $\tau$ axis", were explicitly stated. Therefore, the RBC epistemic actions of the construction stage of the AiC construct were well-performed by Geofrey because he could recognize (R) relevant pieces of knowledge: tangent, velocity, $\tau$ axis which Geofrey reflected on in order to build (B) the definition of the notion of angle of contiguity.

Another example belonging to the AD category was produced by Alice who wrote:

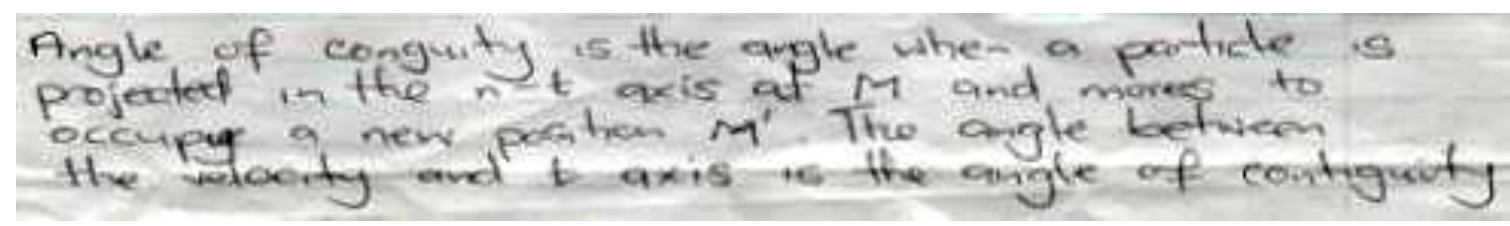

Figure 3. Alice's concept definition

Alices response has similar characteristics to Geofreys personal concept definition previously discussed. A slight distinction between the two student teachers productions is that Geofrey used the correct symbol $\Delta \theta$ for the angle of contiguity which is not evident in Alices personal definition. Furthermore, Alice produced correct graphical illustration. Hence, her responses were more revealing as there was consistency between the narrative and the graphical instantiation of the focal idea. In other words, the two responses 
revealed that the meaning of the concept was invariant under a change in representation from narrative to visual representation of the same idea (Zaslavsky \& Shir, 2005). Overall, the fact that only $25 \%$ ( 8 out of 32 ) of students responses could be classified as adequate is a cause for concern given that the prospective teachers would be expected to competently teach Kinematics concepts at high school level after undergoing the CPD programme of study.

\section{Category: Ambiguous (AMB) student personal concept definitions}

Students written productions in this category consisted of personal concept definitions which could not be uniquely interpreted and were somewhat vague. Typical exemplifications are now produced. First, Modesters personal concept definition is examined.

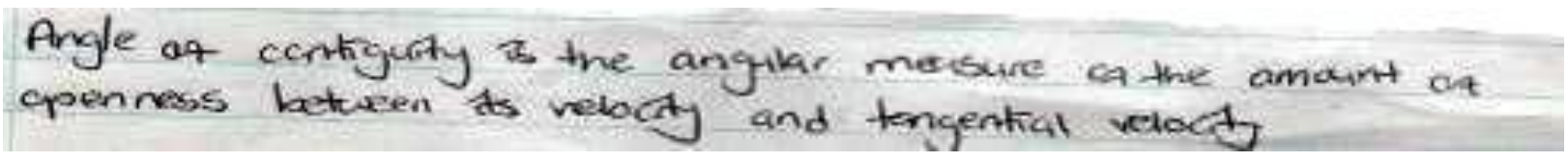

Figure 4. Modesters concept definition

The ambiguity in Modesters response is illuminated by her description of the angle of contiguity as "the openness between its velocity and tangential velocity." This is a vague formulation since the velocity at any instant must be a tangent to the curve at that particular point on the curve. The personal concept definition by Modester reveals that there was lack of coherence in her hierarchical order of knowledge elements that were supposed to constitute the definition of the concept. In other words, she demonstrated a fragile grasp of knowledge items needed to construct the definition of the angle of contiguity. Furthermore, Modester's attempt to construct the definition revealed deficiencies in her epistemic states (Gilboa et al., 2019), because she failed to recognize that velocity must be a tangent to the curve. She claimed that $\Delta \theta$ is the angle between "the tangential velocity and the velocity."

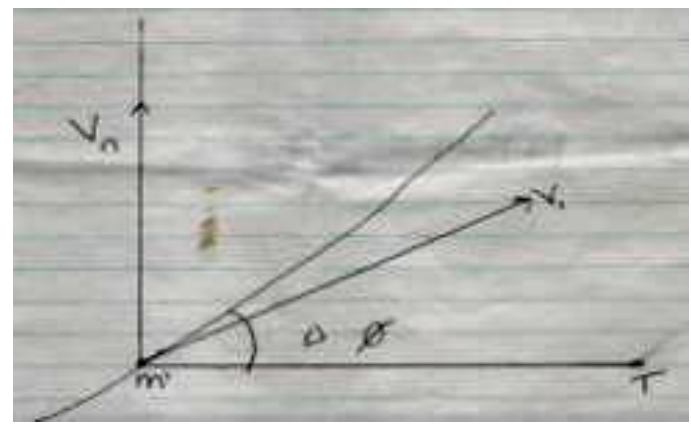

Figure 5. Modesters graphical illustration of the concept of angle of contiguity

Gilboa et al. define a mathematical definition as a meta-mathematical term which serves to indicate the status and function of the concept being considered. From Figure 5, it can be seen that Modesters graphical illustration does not correctly reveal the status of the notion of angle of contiguity since Figure 2 conveys $\delta s$ as the angle the curve (path) makes with the horizontal axis instead of being the angle the velocity vector makes with the tangential axis at $M^{\prime}$. 
Another example of an ambiguous personal concept definition of the angle of contiguity produced by Rashiwe is now presented.

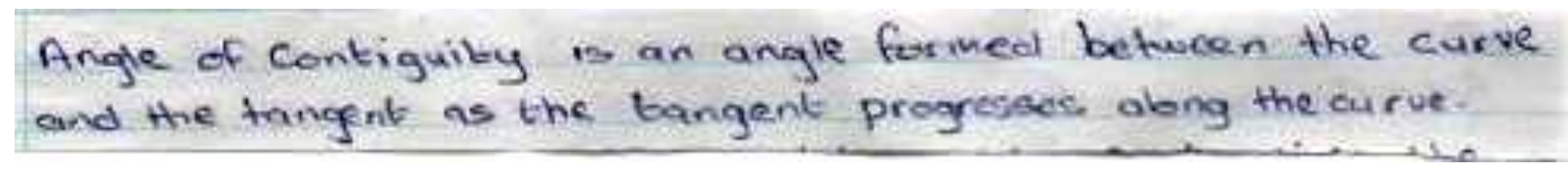

Figure 6. Rashiwes concept definition

It can be noted that the statement by Rashiwe contradicts the definition of the notion of the angle of contiguity because she was supposed to describe it as the angle between the velocity and the horizontal axis. Not only did Rashiwe apply an incorrect epistemic action but she also failed to recognize pieces of knowledge needed to construct the definition namely the need for a tangent as representing the velocity and the horizontal axis. The statement by Rashiwe is ambiguous in the sense that it does not specify whether it refers to the angle on the right or the left of the point of contact between the curve and the tangent. Hence, the statement describing the angle of contiguity as "angle formed between the curve and the tangent" cannot be uniquely interpreted (Zaslavsky \& Shir, 2005). Furthermore, the phrase "as the tangent progresses along the curve" indicates that the tangent has contact with the curve at more than one point - a violation of the restriction imposed on the condition a straight line to a tangent to a curve (Gilboa et al., 2019). Overall, the fact that there were six cases of ambiguous productions by student teachers is a cause for serious considerations in the teaching and learning of Theoretical Mechanics concepts dependent on the concept of the angle of contiguity.

\section{Category: Invariant under change of representation system (IUCR)}

This category consists of responses that conveyed different meanings between narrative and graphical forms of student teachers written attempts. A typical response in this category was produced by Tinei.

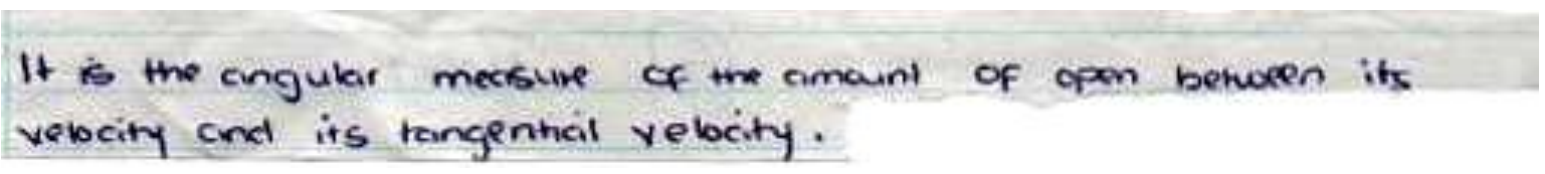

Figure 7. Tinei's concept definition

Tinei's definition of the concept as "the amount of open between its velocity and tangential velocity" is the same as that produced by Modester and hence it is also ambiguous. One of the imperative features of a mathematical definition is that it must be invariant under different systems of representation of that particular concept. However, while Tinei's narrative form of the personal concept definition was ambiguous, he went on to produce a correct graphical illustration of the focal idea. Hence, meaning was not invariant under a change of modes representation. Another case in point of an ambiguous formulation was produced by Oscar who wrote:

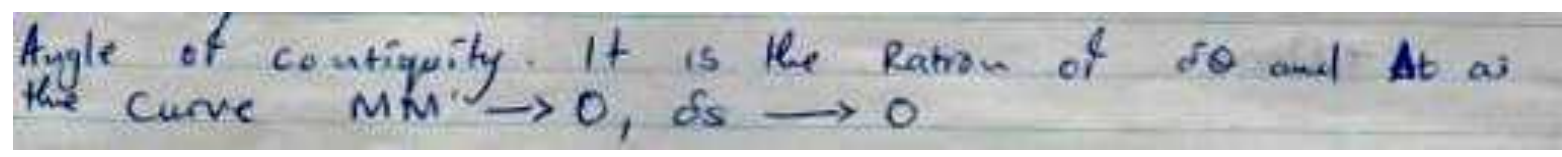

Figure 8. Oscars concept definition 
Oscar's effort can be explained in terms of the epistemic actions of AiC theoretical framework. Thus, while Oscar was able to recognize $(\mathrm{R})$ the basic elements of knowledge relevant for the construction of the definition such as the idea of arc $M M^{\prime}$. Furthermore, Oscar also made the crucial observation that $\operatorname{arc} M M^{\prime}$ must be an infinitesimal displacement by noting that, $\delta s \rightarrow 0$. However, it was at the building (B) epistemic level whereby Oscar could not establish the correct relationship between the basic elements identified. Precisely, Oscar was supposed to introduce the idea of a tangent to the curve and was then supposed to define the angle of contiguity as the angle between the velocity (tangent) and the horizontal axis. In stark contrast to the narrative form, Oscar's visual representation of angle contiguity was correct. Yet, meaning of a concept must be invariant within systems of representation of the same idea as suggested by (Zaslavsky \& Shir, 2005)

Finally, Slyvia described the angle of contiguity as:

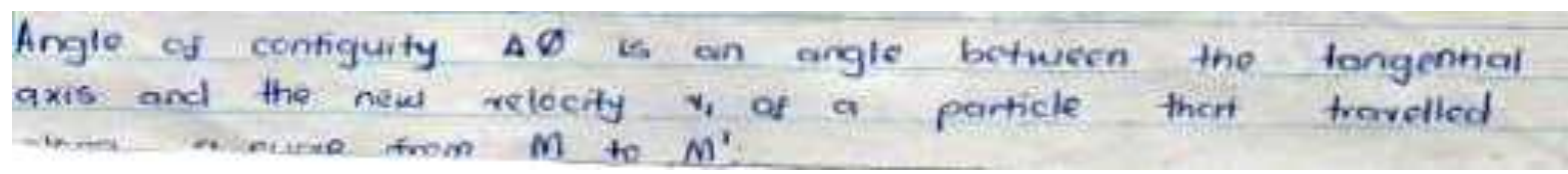

Figure 9. Slyvia's concept definition

While Slyvia's personal concept definition can be classified as adequate, her visual representation of the angle of contiguity did not show the velocity at $M^{\prime}$ as a tangent to the curve as anticipated. Hence, the meaning of the concept was not invariant as she switched from narrative to graphical instantiation of the idea.

\section{Categories: OTHER and Blank (B)}

We now discuss responses categorized as "OTHER" and finally reports on cases where student teachers did not produce written evidence of their concept definitions. The category designated as "OTHER" comprised responses which could not be explained in terms of imperative features of a mathematical definition and epistemic actions of the AiC construct. A typical example of a written response in this category was produced by Farai.

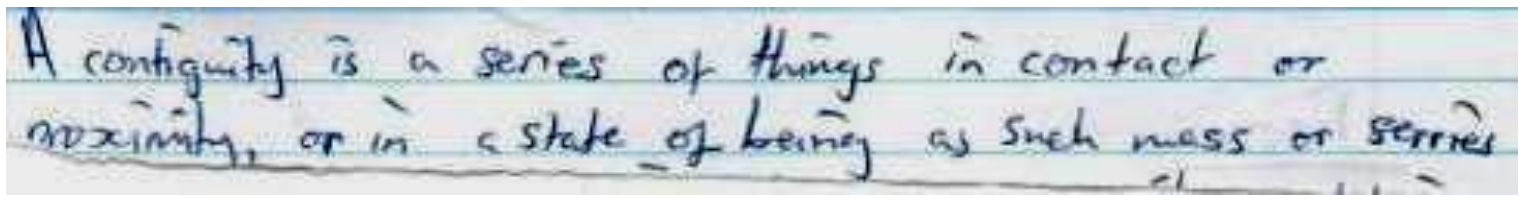

Figure 10. Farai's concept definition

Farai's personal concept definition is an awkward formulation composed of irrelevant ideas such as "state of being ...mass." The concept of mass is irrelevant to Kinematics, a branch of Mechanics that deals with geometrical properties of motion of bodies regardless of their inertial properties which embrace the notion of mass. Farai's claim that the concept of angle of contiguity "is a series of things in contact or in proximity", reveals that he could not recognize (R) the basic knowledge elements required to construct the definition of the concept of angle of contiguity. It can, therefore, be inferred that Farai had no grasp of the focal idea despite that he had gone through the Mechanics module during the 15 week long semester - a cause for concern given that Farai would be expected to 
teach Kinematics concepts to high school students upon completion of undergraduate studies.

Another student, Maureen defined the angle of contiguity as:

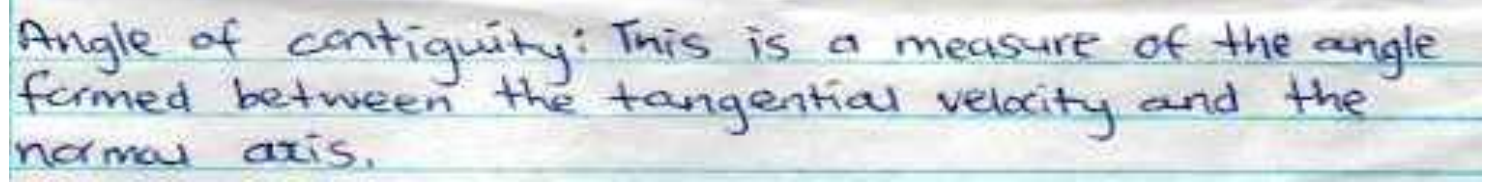

Figure 11. Maureen's concept definition

Maureen's written effort is different from the case of Farai. While Maureen could recognize basic relevant ideas about tangential velocity but she went to refer to an irrelevant idea, that is, normal axis. The term "tangential velocity" is not minimal because velocity of a particle implies that the idea of being a tangent was already embedded. Furthermore, the angle between the "velocity and the normal axis" actually denotes the compliment of the angle of contiguity. Hence, similar to Farai's personal concept definition, Maureen's personal concept definition revealed that she had no sense of the focal idea. In other words Maureen was unable to use existing knowledge elements strategically as also reported by Miyazaki, Fujita and Jones (릭).

Another irrelevant formulation of the idea was produced by Nathan who wrote that the angle of contiguity "refers to adjacent sides and therefore the angle between the two adjacent sides." Similar to Maureen and Farai's responses discussed previously in this subsection, Nathan's description of the concept as "the angle between two adjacent sides" reveals that he had no sense of the angle of contiguity. In other words, Nathan could not access the basic knowledge pieces namely: tangent to the path, tangential axis, normal axis and differential displacement element, $\delta s$ during an infinitesimal time element, $\delta t$. These pre-requisite ideas were not within Nathans conceptual reach and hence the weird answer generated. Data analysis revealed that 4 out of 32 responses were irrelevant formulations. In addition, 6 student teachers did not write their personal concept definitions and this category was called "Blank" (B). Once again, the inconsistent descriptions by Nathan, Maureen and Farai and the $6 \mathrm{~B}$ responses point to severe limitations in undergraduate students abilities to construct definitions.

\section{Conclusions}

The discussion of results has led to the following conclusions about the kinds of student teachers personal concept definitions of the notion of the angle of contiguity in Kinematics. Overall, students demonstrated a weak grasp of the concept of angle of contiguity since only 8 out of 32 students could produce adequate definitions of the concept and a significant number ( 6 out of 32 ) could not construct the definition of the term. From the discussion of the individual categories, it could be inferred that students' conceptions of the concept could be characterized as follows.

First, undergraduate mathematics education students' efforts revealed a good grasp of the fundamental ideas needed to compose the definition. For instance, they showed an appreciation of the idea that velocity is a tangent to the curve. Furthermore, informants' efforts revealed that the notation involved the use of symbols such as $\Delta \theta$ to denote an 
infinitesimal change in the angle (e.g. the case of Geofrey). Hence, it can be concluded that the $(\mathrm{R})$ epistemic state of the AiC construct had been attained. Furthermore, descriptions such as "as the curve, $M M^{\prime} \rightarrow 0, \delta s \rightarrow 0$ " reinforce the idea that students had an appreciation of the fact that the concept of angle of contiguity involves infinitesimal quantities - a crucial idea in their efforts to construct the definition. Hence, mathematicians and mathematics educators need to reinforce these basic ideas in mathematics instruction.

Second, it can be concluded that most students had not reached the building (B) epistemic level of the AiC construct because they failed to relate prerequisite ideas needed to construct the definition of the notion of angle of contiguity. So it can be inferred that a gap existed between the $\mathrm{R}$ and $\mathrm{B}$ epistemic levels that characterize their conceptions of the idea. This study has shown that participants' personal concept definitions were characterized by ambiguous formulations which could not be uniquely interpreted. These can be attributed to the disconnection alluded to between the $\mathrm{R}$ and $\mathrm{B}$ epistemic levels in their conceptions of the notion of angle of contiguity. Hence, there is need for mathematics educators and instructors at undergraduate level to devise means aimed at bridging the gap between the $\mathrm{R}$ and $\mathrm{B}$ epistemic levels when constructing mathematical definitions.

Third, it can also be inferred from the Results and Discussion Section that the kinds of students' constructions were characterized by weird and irrelevant formulations such as a "series of things in contact or proximity or in a state of mass" (referring to Farai's written effort). Such attempts illustrate that they lacked prerequisite ideas and hence, they were probably resorting to guess work because they had no essence of the focal mathematical ideas. It is, therefore, recommended that mathematics instructors seriously consider introducing a module covering basic mathematical ideas instead of treating such pieces of knowledge as assumed knowledge. Furthermore, some student teachers productions were contradicting the concept definition of the angle of contiguity. In addition, the fact that there was a significant number of responses ( 6 out of 32 ) in the category labelled "blank" (B) that denotes cases whereby students could not define the concept of angle of contiguity revealed severe limitations in students' abilities to construct mathematical definitions. These cases also deserve serious attention when devising mathematics instruction.

Fourth, students written productions revealed inconsistencies in the form of different meanings conveyed by narrative descriptions and visual representations of the focal mathematical idea. In other words, the study revealed lack of coherence between the graphical illustrations and the narrative forms of the mathematical definitions. Therefore, students' personal concept definitions responded to a switch from verbal descriptions to graphical instantiations. These inconsistencies were indicative of severe limitations in students' conceptual knowledge. This finding has serious consequences for mathematics instructors who should strive to enhance students' conceptual understanding of mathematical concepts. Improved conceptual understanding will allow students to switch from one form of representation to another without distortions in the meaning of the concept.

The inadequacies revealed by this study with respect to undergraduate students' grasp of the notion of angle of contiguity deserve serious consideration by mathematics educators and research mathematicians in order to improve the teaching and learning of 
the concept and those concepts dependent on it. Exploring the consolidation stage of the $\mathrm{AiC}$ construct can assist in this regard. In other words, research studies on students' abilities to use the concept of angle of contiguity in problem solving and proof activities may help foster improved conceptions of the idea and ameliorate limitations uncovered by the current study. This study corroborates efforts by mathematics education researchers to promote and foster enhanced conceptualizations of mathematics concepts among learners. By revealing the kinds of disparities between the definition of the angle and informants productions, the study has generated ideas that have the potential to inform mathematics instruction.

\section{Acknowledgment}

We express our gratitude to our employers for granting us study leave that made it possible to work on this paper. We also sincerely thank the informants for cooperation during data collection.

\section{Bibliography}

Baxter, P., \& Jack, S. (2008). Qualitative case study methodology: Study design and implementation for novice research. The Research Report, 13(4), 544-559.

Berg, B.L. (2009). Qualitative research methods for the social sciences Topics. Boston: Allyn Brown.

CadawalladerOlsker, T. (2011). What do we mean by mathematical proof. Journal of humanistic mathematics, 1(1), 1-33.

Carlson, M.P., Bloom, I. (2005). The cyclic nature of problem solving: an emergent multidimensional problem-solving-framework. Educational Studies in Mathematics, $58,58-75$.

Corbin, J., \& Strauss, A. (2008). Basics of qualitative research. Thousand Oaks: Sage.

Creswell, J.W. (2014). Research design: Qualitative, quantitative and mixed methods approaches. London: Sage.

Davydov, V.V. (1990). Types of generalization in instruction: Logical and psychological problems in structuring school curricula. In J. Patrick (Ed), Soviet Studies in Mathematics Education. Reston: National Council of Teachers for Mathematics (NCTM).

Dreyfus, T., Hoyles, C., Gueudet, G., \& Krainer, K. (2014). Solid findings: concept images in students mathematical reasoning. European Mathematical Society Newsletter.

Edwards, B., \& Ward, M.B. (2004). Surprises from mathematics education research: students (mis) use of mathematical definitions. American Mathematical Monthly, 411424.

Gilboa, N., Kidron,I., \& Dreyfus, T. (2019). Constructing a mathematical definition: the case of the tangent. International Journal of Mathematics Education in Science and Technology, 50(3), 421-436. https://doi.org/10.1080/0020739X.2018.1516824

Harel, G., \& Sowder, L. (1998). Students proof schemes: Results from exploratory studies. In Schoenfeld, A., Kaput, J., and Dubinsky, E., (Eds.), Research in Collegiate Mathematics Education: Vol. 3. (pp. 234-282). Washington, DC: American Mathematical Society.

Jamillah, Suryadi, D., \& Priatna, N. (2019). Students concept images on set: zone of differences between concept image and formal concept definition. International Journal of Advanced Science and Technology, 28(18), 156-166. 
Kidron, I. (2011). Constructing knowledge about the notion of limit in the definition of the horizontal asymptote. International Journal of Science and Mathematics Education, 9, 1261-1279. https://doi.org/10.1007/s10763-010-9258-8

Maxwell, J.A., \& Mittapalli, K. (2010). Realism as a stance for mixed methods research. In A. Tashakkori and T. Teddlie (Eds), Sage handbook of mixed methods in social behavioural research (pp.145-166). Washington DC: Sage Publications.

Mariotti, M.A., \& Fischbein, E. (1997). Defining in classroom activities. Educational Studies in Mathematics, 34, 58-69. https://doi.org/10.1023/A:1002985109323

Miyazaki, M., Fujita, T., \& Jones, K. (2017). Students understanding of the structure of a deductive proof. Educational Studies in Mathematics, 94, 223-239. https://doi.org/10.1007/s10649-016-9720-9

Nurwahyu, B. (2014). Concept image definition of students concept understanding. In M. A., Futhani and T. Permadi (Eds), Proceedings of the International Seminar on Mathematics Education and Graph Theory, Islamic University of Malang: Malang.

Pimm, D. (1993). Just a matter of a definition. Educational Studies in Mathematics, 25, 261277.

Punch, K. F. (2005). Introduction to social science research: Quantitative and qualitative approaches. London: Sage.

Schoenfeld, A.H. (1982). Some thoughts on problem solving research and mathematics education. In F.K. Lester \& J. Garofalo (Eds.), Mathematical problem solving: Issues in Research. Franklin Institute Press, Pennsylvania, pp. 27-37.

Selden, A., \& Selden, J. (2003). Validations of proofs considered as texts: Can undergraduates tell whether an argument proves a theorem? Journal forResearch in Mathematics Education, 34, 4-36.

Ubuz, B., Dincer, \& Bülbül, A. (2013). Argumentation in undergraduate math courses: A study on definition construction. In A.M. Lindmeier, \& A. Heinze (Eds.), Proceedings of the 37th Conference of International Group for the Psychology of Mathematics Education: Vol. 4. (pp. 313-320). Kiel University: PME.

VanDormolen, J., \& Zaslavsky, 0. (2003). The many facets of a definition: the case of a definition: The case of periodicity. Journal of Mathematical Behaviour, 22, 91-106. https://doi.org/10.1016/S0732-3123(03)00006-3

Vinner, S. (1991). The role of definitions in the teaching and learning of mathematics. In D. Tall (Ed), Advanced mathematical thinking. Dordrecht (The Netherlands): Kluwer, $65-81$.

Vinner, S., \& Hershwitz, R. (1980). Concept images and common cognitive paths in the development of some simple geometric concepts. In R. Karplus (Ed.), Proceedings of the $4^{\text {th }}$ Conference of the International Group for the Psychology of Mathematics Education: (pp. 177-184). Berkeley, CA: PME.

Wilkerson-Jerde, M., \& Wilensky, U.J. (2011). How do mathematicians learn math?: resources and acts for constructing and understanding mathematics. Educational Studies in Mathematics, 78, 21-43.

Wilson P. S. (1990). Inconsistent ideas related to definitions and examples. Focus on Learning Problems in Mathematics, 12(3-4), 31-47.

Yin, R. K. (2009). Case study research: design and methods. Thousand Oaks: Sage.

Zaslavsky, O., \& Shir, K. (2005). Students conceptions mathematical definition. Journal for Research in Mathematics Education, 36 (4), 317-346. https://doi.org/10.2307/30035043 